This is the post print version of the article, which has been published in Laryngoscope. 2018, 128(9), 1997-2003.https://doi.org/10.1002/lary.27103.

\title{
A Prospective, Randomized, Placebo-Controlled Study of Inferior Turbinate Surgery
}

Teemu Harju, MD; Jura Numminen, MD, PhD; Ilkka Kivekäs, MD, PhD; Markus

Rautiainen, MD, PhD

The This document has been downloaded from TamPubuta.fi

From the Department of Otorhinolaryngology, Tampere University Hospital, Tampere,

Finland

Running title: Placebo-controlled study of turbinate surgery

Conflicts of interest: None

Financial disclosures: The authors received no financial support for the research, authorship, and/or publication of this article.

\section{Corresponding author:}

Teemu Harju, MD, Department of Otorhinolaryngology, Tampere University Hospital,

Teiskontie 35, 33521 Tampere, Finland

E-mail: harjtee@gmail.com 


\section{ABSTRACT}

Objectives: The purpose of this prospective, randomized, single-blinded, placebo-controlled study is to compare radiofrequency ablation, diode laser, and microdebrider-assisted inferior turbinoplasty techniques in the treatment of chronic nasal obstruction caused by inferior turbinate enlargement, and to compare these techniques with a placebo procedure.

Methods: A total of 98 consecutive patients with enlarged inferior turbinates due to persistent year-round rhinitis were randomized into a placebo, radiofrequency ablation, diode laser, and microdebrider-assisted inferior turbinoplasty groups in a ratio of 1:2:2:2. All the procedures were carried out under local anesthesia with the patients' eyes covered. Assessments were conducted prior to surgery and three months subsequent to the surgery.

Results: The severity of nasal obstruction measured by VAS score decreased statistically significantly in all the groups, including placebo. Radiofrequency ablation $(p=0.03)$, diode laser $(p=0.02)$, and microdebrider-assisted inferior turbinoplasty $(p=0.04)$ all decreased the symptom score of the severity of nasal obstruction statistically significantly more compared to the placebo procedure.

Conclusion: The placebo effect had a large role in the overall improvement of the severity of nasal obstruction after the inferior turbinate surgery. However, all three techniques provided a statistically significant additional reduction of the severity of nasal obstruction compared to the placebo procedure.

Key Words: Nasal obstruction, inferior turbinate surgery, placebo-controlled, randomized 


\section{INTRODUCTION}

Inferior turbinate enlargement due to persistent year-round allergic or non-allergic rhinitis is one of the main causes of chronic nasal obstruction. ${ }^{1}$ Intranasal corticosteroids are the most important medicine group in the treatment of rhinitis and hypertrophied inferior turbinates. When medical therapy for enlarged inferior turbinates fails, turbinate surgery can be considered. ${ }^{2}$

Various surgical techniques have been described for the reduction of hyperplastic inferior turbinates. However, no clear consensus exists in the literature on the exact role of surgery or the most optimal method for surgical treatment. ${ }^{3}$ In recent years, surgical procedures have concentrated on the minimal disturbance of the nasal mucosa. The aim of inferior turbinate surgery has been to maximize the volumetric reduction of the turbinate to decrease the nasal obstruction while maintaining nasal function and minimizing complications. ${ }^{4}$

Microdebrider-assisted inferior turbinoplasty (MAIT) - a powered subtype of submucosal resection $^{5}$ - and radiofrequency ablation (RFA) are referred to as mucosal sparing techniques; they are widely used and the most commonly studied techniques in the recent literature. ${ }^{4}$ The diode laser technique has also gained in popularity due to its ease of use in the office setting. ${ }^{6}$

Thus far, three placebo-controlled trials have been published regarding RFA. These studies have evaluated the influence of the treatment on subjective scores of nasal obstruction, and they found that the placebo effect seems to have a role in the results of turbinate surgery. ${ }^{7,8,9}$ 
The purpose of this prospective, randomized, single-blinded, placebo-controlled study is to compare RFA, diode laser, and MAIT techniques in the treatment of chronic nasal obstruction caused by inferior turbinate enlargement, and to compare these techniques with a placebo treatment.

\section{METHODS}

This prospective randomized study was carried out at Tampere University Hospital, Tampere, Finland, between February 2014 and September 2017. The institutional review board approved the study design (R13144) and all patients provided written, informed consent. A total of 98 consecutive adult patients with enlarged inferior turbinates due to persistent year-round rhinitis were enrolled in this study. The patients presented symptoms of bilateral nasal obstruction related to inferior turbinate congestion that had not responded to a three-month trial of appropriate treatment with intranasal corticosteroids. Patients with significant nasal septum deviation affecting the nasal valve region, internal/external valve collapse/stenosis, chronic rhinosinusitis with or without polyposis, previous nasal surgery, sinonasal tumor, severe systemic disorder, severe obesity, or malignancy were excluded.

Cone beam computed tomography (Planmeca Max, Planmeca, Helsinki, Finland) was used to exclude patients with chronic rhinosinusitis from the study. Serum-specific IgE level measurements were used to identify the patients with an allergic sensitization. Allergic sensitization was defined as a specific $\lg \mathrm{E}>0.35$ for any common airborne allergen (cat, dog, horse, birch, grass, mugwort, D. pteronyssinus, and molds). 
The definition of inferior turbinate enlargement was based on persistent bilateral symptoms, a finding of bilateral swelling of the inferior turbinate in nasal endoscopy, and the evident shrinking of both turbinates in a decongestion test, which could not be applied to either bony turbinate enlargement of soft tissue hypertrophy unresponsive to topical decongestant. The nasal response to the topical vasoconstrictor $0.5 \%$ xylometazoline hydrochloride (Nasolin, Orion, Finland) in both nasal cavities 15 minutes before obtaining the second measurement was evaluated objectively using acoustic rhinometry (Acoustic rhinometer A1, GM instruments Ltd, Kilwinning, UK). An improvement of less than $30 \%$ in anterior nasal cavity volume ( $\mathrm{V} 2-5 \mathrm{~cm})$ in one or both nasal cavities was considered normal and those patients were excluded from the study. The limit value of $30 \%$ was chosen according to previous literature. ${ }^{10,11,12}$

Patients were consecutively randomized into placebo, RFA, diode laser, and MAIT groups in a ratio of 1:2:2:2 using Minim, a free MS-DOS program that randomizes patients to treatment groups by the method of minimization. Proportional amounts of patients with allergic sensitization were kept similar for each group. Age and sex distributions were also kept similar for each group.

The surgical treatment was performed in similar circumstances at the day surgery department of the hospital's ENT clinic. All surgical procedures were performed by the same surgeon (T.H). The staff of the day surgery department was instructed to avoid all communication before and during the operation, as well as during the post-operative care, that might reveal the group of the patient. All the alternative surgical devices were available in the operation room. The procedures were carried out under local anesthesia with the patient's eyes covered. 
First, the inferior turbinate was topically anesthetized using cotton strips with a mixture of lidocaine 40mg/ml (Lidocain, Orion, Finland) and 2-3 drops of epinephrine $0.1 \%$ in 5-10 $\mathrm{ml}$ of lidocaine. $1.5 \mathrm{ml}$ of local anesthetic (Lidocain $10 \mathrm{mg} / \mathrm{ml} \mathrm{c}$. adrenalin $10 \mu \mathrm{g} / \mathrm{ml}$, Orion, Finland) was then applied to the medial portions of both inferior turbinates. All the procedures were performed under the direct vision of a straight, $4 \mathrm{~mm}$-diameter, 0-degree endoscope (Karl Storz, Germany). In all the groups with every technique, the treatment was given to the medial side of the anterior half of the inferior turbinate. Short-term (until next morning) nasal packing with non-absorbable packing material (Ivalon, Fabco, New London, Connecticut) was applied only if active bleeding developed during the surgery.

The RFA treatment was carried out with a radiofrequency generator (Sutter RF generator BM-780 II). A "Binner" bipolar needle electrode was inserted into the medial submucosal tissue of the inferior turbinate. The upper and lower parts of the anterior half of the inferior turbinate were treated for $6 \mathrm{sec}$ at $10 \mathrm{~W}$ output power in three areas.

The diode laser treatment was given with a FOX Laser (A.R.C. LASER GmbH, Nuremberg, Germany). The settings were as follows: wavelength of $980 \mathrm{~nm}$, output power of $6 \mathrm{~W}$ in continuous-wave mode, and laser delivery by a $600 \mu \mathrm{m}$ fiber using "contact" mode. Four parallel stripes were made on the mucosa by drawing the fiber from the posterior to the anterior direction along the medial edge of the anterior half of the inferior turbinate.

In the MAIT treatment, a $2.9 \mathrm{~mm}$-diameter rotatable microdebrider tip (Medtronic Xomed, Jacksonville, Florida) was firmly pushed toward the turbinate bone until it pierced the 
mucosa of the anterior face of the inferior turbinate. Next, a submucosal pocket was dissected by tunneling the elevator tip in an anterior-to-posterior and superior-to-inferior sweeping motion. Once an adequate pocket had been created, resection of the stromal tissue was carried out by moving the blade back and forth in a sweeping motion, with the system set at $3000 \mathrm{rpm}$ using suction irrigation.

In the placebo procedure, small (2-3 $\mathrm{mm}$ in diameter) nasal mucosal biopsies were first taken from the anterior medial portions of the inferior turbinates, causing minor bleeding. Next, a radiofrequency tissue ablation device was turned on repeatedly near the patient, but without the needle electrodes of the device touching the patient; the patient could only hear the acoustic tone of the device. During this sound deception, a suction tube and a nasal endoscope were moved lightly in both sides of the nose for a couple of minutes in order to convince the patients that they had undergone surgery.

After the operation, none of the patients were given medical treatment, including analgesics, nasal steroids and nasal decongestants. The patients were given a $100 \mathrm{~mm}$ visual analogue scale (VAS) questionnaire, which had been filled for the first time during the preoperative visit. They were asked to mark on the line the point that they feel represents their perception of their current state regarding post-operative nasal symptoms, including severity of nasal obstruction (not obstructed - very obstructed), discharge (no discharge - much discharge), crusting (no crust - much crust), and pain (no pain - much pain). The questionnaire was to be filled daily for the first week, and then after two, three and four weeks post-operatively. They were instructed to return the questionnaire by post one month after the operation. 
All the patients were evaluated prior to surgery and three months subsequent to the surgery. All clinical examinations were performed by the same examiner (T.H) who was also the operator and not blinded to the patients' group. The patients filled the VAS questionnaire regarding nasal symptoms during both visits. They were asked to mark on the line the point that they feel represents their perception of their current state. Acoustic rhinometry was also performed both before the surgery and during the control visit at three months. During the control visit, the patients filled the questionnaire before meeting the examiner.

IBM SPSS Statistics 22.0 was used for the statistical analyses. All non-parametric data were statistically processed using the Wilcoxon signed-rank test, Mann-Whitney $U$ test, and Kruskal-Wallis test. In the cases with parametric data, the comparison between the groups was carried out by one-way ANOVA.

\section{RESULTS}

The patients' characteristics are described in Table 1 and the data of the operations in Table 2. The operation time for the MAIT procedure was statistically significantly longer compared to the other procedures. The operation time for the diode laser, in turn, was statistically significantly longer than the time for the RFA. The VAS score for pain during the operation was highest in the MAIT group, but there were no statistically significant differences between the groups. Five patients in the MAIT group and one patient in the diode laser group had moderate bleeding that required short-term (until next morning) nasal packing. Four patients in the MAIT group complained of notable bleeding during the 
first post-operative day and three of them had to be treated at the outpatient clinic with short-term (until next morning) nasal packing.

The changes in nasal symptoms reported by the patients after the operation are described in Figure $1(A-D)$. The severity of nasal obstruction started to decrease immediately after MAIT and continued up to four weeks. In the diode laser and RFA groups, the response appeared slightly slower. In the placebo group, the response also started immediately and reached its final level after two weeks.

At the control visit at three months, minor crusting was seen in the nasal cavities of a single patient in the placebo group, in 12 patients in the RFA group, in six patients in the diode laser group, and in two patients in the MAIT group. One patient in the MAIT group had a small synechia. Atrophy was not found in any of the cases.

The changes in the VAS scores for nasal symptoms at three months are described in Table 3. The severity of nasal obstruction decreased statistically significantly in all the groups. All the techniques used were compared separately with placebo using the MannWhitney $U$ test. RFA $(p=0.02)$, diode laser $(p=0.03)$, and MAIT $(p=0.04)$ all decreased the symptom score for the severity of nasal obstruction statistically significantly more compared to the placebo procedure.

In all the groups, nasal discharge and crusting decreased statistically significantly, but there were no statistically significant differences between the groups, including the placebo group (Table 3). 
The change in V2-5 $\mathrm{cm}$ acoustic rhinometry values is described in Table 4 . All three techniques improved the $\mathrm{V} 2-5 \mathrm{~cm}$ values significantly, but there were no statistically significant differences between the groups, including placebo.

\section{DISCUSSION}

There are studies reporting notable intraoperative bleeding ${ }^{13}$ or post-operative bleeding in the early days after MAIT, ${ }^{14}$ and this was also the case in the present study. During the operation, the mean VAS score for pain was the highest in the MAIT group, but there was no statistical difference between the groups. Post-operatively, there was an increase in the VAS score for pain in the MAIT group, which lasted up to one week. This can possibly be explained by the direct surgical tissue trauma caused by the operation. Short-term nasal packing, which was most common in the MAIT group, may also partly explain the increase of pain during the first days of the follow-up. Increased pain was also reported during the first two days after diode laser treatment. This can possibly be explained by the higher temperature and therefore the more severe tissue damage caused by the laser compared to the RFA. In the present study, a temporary increase in the symptom of nasal discharge and a minor increase in the symptom of crusting during the first post-operative days after RFA was noted. A similar finding has been reported in the previous studies. ${ }^{15,16}$ Some patients treated with RFA complained of crust formation for as long as three months. In a study by Janda et al., patients showed moderate-to-severe nasal obstruction, crusting, and nasal secretion in the first four weeks following diode laser treatment. ${ }^{6}$ In the present study, however, patients in the diode laser group on average reported a decrease in crusting immediately after the operation and an increase in nasal discharge only during the first two days. In some cases, however, crust formation continued up to three months. 
In recent years, studies have been published comparing RFA and MAIT in the treatment of nasal obstruction. There are studies where the results of MAIT have been better in the short follow-up ${ }^{14}$ and also in the longer follow-up. ${ }^{17}$ There are also studies where the techniques have been found equal in efficacy. ${ }^{18}$ A systematic review and meta-analysis by Acavedo et al. compared RFA and MAIT. They concluded that inferior turbinate reduction produces a significant subjective and objective improvement in nasal airflow in the short term, which is not related to the technique used. ${ }^{19}$ There are also studies comparing RFA and the diode laser ${ }^{20,21}$ and MAIT and the diode laser, ${ }^{13}$ and no significant differences between the techniques have been found in any of these studies. No previous studies have compared all three techniques together.

In the present study, the decrease in the VAS score for severity of nasal obstruction started faster after MAIT. In MAIT, the submucosa of the turbinate is emptied immediately during the operation, whereas in the RFA and diode laser procedures, the heat caused by the device creates a submucosal lesion, inducing scarring and tissue volume reduction during the following weeks. ${ }^{1,22}$ Post-operative nasal packing, which was most common in the MAIT group, squeezes the mucosa of the emptied turbinate against the bone and may so contribute to the faster response. Based on this conclusion and the higher rate of postoperative bleeding in the MAIT group, nasal packing can be recommended in the MAIT procedures. At the end of the three-month follow-up, all three techniques decreased the VAS score for the severity of nasal obstruction and V2-5 cm values statistically significantly compared to the preoperative values, and they were equal in efficacy regarding both parameters. 
So far, there have been only three placebo-controlled trials evaluating the influence of the treatment on the subjective scores of nasal obstruction. In a double-blinded, placebo-controlled trial by Powell et al., 22 patients were randomly assigned to either an RFA ( $n=17)$ or placebo $(n=5)$ group. The beneficial effect of treatment on nasal obstruction with RFA was demonstrated, but it did not reach statistical significance, probably due to the small sample size. ${ }^{7}$ A single-blinded, placebo-controlled trial with a crossover option by Nease et al. included 32 patients who were randomized to either an RFA ( $n=16)$ or placebo $(n=16)$ group. Improvement was shown in the frequency of nasal obstruction, severity of nasal obstruction, and overall ability to breathe for both the placebo and RFA treatment arms at 8 weeks. However, the effects of RFA were significantly better than those of placebo in the severity of nasal obstruction and overall ability to breathe. ${ }^{8}$ In a single-blinded, placebo-controlled, crossover trial by Bran et al. with 22 patients, the first group received RFA first followed by a placebo treatment 6-8 weeks later. In the second group, the order was reversed. Nasal obstruction decreased significantly only after RFA in both groups. There was a tendency of improvement after placebo in both treatment arms. However, the observed trends of a placebo effect were not statistically significant. ${ }^{9}$

Our study is the first placebo-controlled study involving three different techniques, and also the first study where the diode laser and MAIT have been compared with placebo treatment. The follow-up time of three months with the placebo group is also the longest so far. RFA, diode laser, and MAIT all improved the VAS score for the severity of nasal obstruction statistically significantly better than placebo. The finding is in line with the study by Nease et al. regarding RFA. ${ }^{8}$ The true treatment effect is the difference in improvement between the intervention group and the control group (placebo effect). ${ }^{7}$ In the present study, the mean true treatment effect in the VAS score change was found to be -2.1 for all 
three techniques. The placebo effect accounted for a large part of the total improvement seen in the intervention groups. Without a placebo control, we would have falsely found a greater improvement and significance due to treatment.

In the present study, all the techniques decreased the symptom scores of nasal discharge and crusting statistically significantly compared to the preoperative values at the end of the three-month follow-up, but none of them statistically significantly compared to placebo. In addition, minor crusting was still found in some noses at three months, especially in the RFA and diode laser groups. Therefore, the major part of the improvement of these symptoms can be explained by the placebo effect.

Anterior nasal cavity volume ( $\mathrm{V} 2-5 \mathrm{~cm})$ in acoustic rhinometry was used as a parameter for objective examination. It represents well the operated anterior half of the inferior turbinate. Acoustic rhinometry will give a valid result in most instances, at least for the first $5-6 \mathrm{~cm}$ of the nasal cavity. ${ }^{23}$ Measurement of $\mathrm{V} 2-5 \mathrm{~cm}$ is the most sensitive measurement for change in mucosal swelling during decongestion, ${ }^{24}$ and that is why the parameter also had an important role in the preoperative decongestion test in defining inferior turbinate enlargement. In the three-month follow-up, RFA, diode laser and MAIT techniques all improved the $\mathrm{V} 2-5 \mathrm{~cm}$ values statistically significantly compared to the preoperative values. Due to lack of a real surgical procedure, there was understandably no statistically significant change in the V2-5 cm values in the placebo group. This supports the conclusion that the improvement in the VAS score for severity of nasal obstruction in the placebo group was due to placebo effect. However, none of the techniques improved the volume statistically significantly compared to placebo. This finding can be partly explained by the relatively small number of patients in the placebo group. A larger number might 
have led to a statistical significance. In addition, environmental conditions and technical difficulties in measurement may have an influence in the volume values. ${ }^{25}$ Furthermore, the symptom of nasal obstruction and objective parameters do not always correlate very well. ${ }^{26}$ On the other hand, it is wise to remember that the increase in the nasal cavity volume should be optimal instead of too large in order to avoid making the nose too wide, which might lead to problems such as nasal dryness, crusting, atrophy, worsening of the symptom of nasal obstruction, and "empty nose syndrome".,1,27

\section{CONCLUSION}

After three months' follow-up, there were no statistically significant differences between the examined techniques in their efficacy in treating nasal obstruction. Possible differences in their efficacy may emerge in the long term. Therefore, more studies comparing these techniques with longer follow-ups are needed.

RFA, diode laser, and MAIT were all genuinely effective in treating chronic nasal obstruction caused by inferior turbinate enlargement. The placebo effect had a large role in the overall reduction of the severity of nasal obstruction after the inferior turbinate surgery, but all the three techniques provided a statistically significant additional reduction in the severity of nasal obstruction compared to the placebo procedure.

\section{REFERENCES}

1. Willatt D. The evidence for reducing inferior turbinates. Rhinology 2009;47:227-36. 
2. Rice DH, Kern EB, Marple BF, Mabry RL, Friedman WH. The turbinates in nasal and sinus surgery: a consensus statement. Ear Nose Throat J 2003;82:82-4.

3. Batra PS, Seiden AM, Smith TL. Surgical management of adult inferior turbinate hypertrophy: a systematic review of the evidence. Laryngoscope 2009;119:1819-27.

4. Bhandarkar ND, Smith TL. Outcomes of surgery for inferior turbinate hypertrophy. Curr Opin Otolaryngol Head Neck Surg 2010;18:49-53.

5. Sinno S, Mehta K, Lee ZH, Kidwai S, Saadeh PB, Lee MR. Inferior turbinate hypertrophy in rhinoplasty: Systematic review of surgical techniques. Plast reconstr surg. 2016;138:419e-29e.

6. Janda P, Sroka R, Tauber S, Baumgartner R, Grevers G, Leunig A. Diode laser treatment of hyperplastic inferior nasal turbinates. Lasers Surg Med 2000;27:129-39. 7. Powell NB, Zonato AI, Weaver EM, Li K, Troell R, Riley RW, Guilleminault C. Radiofrequency treatment of turbinate hypertrophy in subjects using continuous positive airway pressure: a randomized, double-blind, placebo-controlled clinical pilot trial. Laryngoscope 2001;111:1783-90.

8. Nease CJ, Krempl GA. Radiofrequency treatment of turbinate hypertrophy: a randomized, blinded, placebo-controlled clinical trial. Otolaryngol Head Neck Surg 2004;130:291-9.

9. Bran GM, Hünnebeck S, Herr RM, Hörmann K, Stuck BA. Bipolar radiofrequency volumetric tissue reduction of the inferior turbinates: evaluation of short-term efficacy in a prospective, randomized, single-blinded, placebo-controlled crossover trial. Eur Arch Otorhinolaryngol 2013;270:595-601.

10. Grymer LF, Hilberg O, Pedersen OF, Rasmussen TR. Acoustic rhinometry: values from adults with subjective normal nasal patency. Rhinology 1991;29:35-47. 
11. Tomkinson A, Eccles R. Comparison of the relative abilities of acoustic rhinometry, rhinomanometry, and the visual analogue scale in detecting chance in the nasal cavity in a healthy adult population. Am J Rhinol 1996;10:161-165.

12. Fisher EW. Acoustic rhinometry. Clin Otolaryngol Allied Sci 1997;22:307-17.

13. Kassab AN, Rifaat M, Madian Y. Comparative study of management of inferior turbinate hypertrophy using turbinoplasty assisted by microdebrider or $980 \mathrm{~nm}$ diode laser. J Laryngol Otol 2012;126:1231-7.

14. Cingi C, Ure B, Cakli H, Ozudogru E. Microdebrider-assisted versus radiofrequencyassisted inferior turbinoplasty: a prospective study with objective and subjective outcome measures. Acta Otorhinolaryngol Ital 2010;30:138-43.

15. Coste $A$, Yona L, Blumen $M$, Louis $B$, Zerah F, Rugina $M$, Peynègre $R$, Harf $A$, Escudier E. Radiofrequency is a safe and effective treatment of turbinate hypertrophy. Laryngoscope 2001;111:894-9.

16. Bäck LJ, Hytönen ML, Malmberg HO, Ylikoski JS. Submucosal bipolar radiofrequency thermal ablation of inferior turbinates: a long-term follow-up with subjective and objective assessment. Laryngoscope 2002;112:1806-12.

17. Liu CM, Tan CD, Lee FP, Lin KN, Huang HM. Microdebrider-assisted versus radiofrequency-assisted inferior turbinoplasty. Laryngoscope 2009;119:414-8. 18. Kizilkaya Z, Ceylan K, Emir H, Yavanoglu A, Unlu I, Samim E, Akagün MC. Comparison of radiofrequency tissue volume reduction and submucosal resection with microdebrider in inferior turbinate hypertrophy. Otolaryngol Head Neck Surg 2008;138:176-81.

19. Acevedo JL, Camacho M, Brietzke SE. Radiofrequency Ablation Turbinoplasty versus Microdebrider-Assisted Turbinoplasty: A Systematic Review and Meta-analysis. Otolaryngol Head Neck Surg 2015;153:951-6. 
20. Rhee CS, Kim DY, Won TB, Lee HJ, Park SW, Kwon TY, Lee CH, Min YG. Changes of nasal function after temperature-controlled radiofrequency tissue volume reduction for the turbinate. Laryngoscope 2001;111:153-8.

21. Kisser U, Stelter K, Gürkov R, Patscheider M, Schrötzlmair F, Bytyci R, AddersonKisser C, Berghaus A, Olzowy B. Diode laser versus radiofrequency treatment of the inferior turbinate - a randomized clinical trial. Rhinology 2014;52:424-30.

22. Cakli H, Cingi C, Güven E, Gurbuz MK, Kaya E. Diode laser treatment of hypertrophic inferior turbinates and evaluation of the results with acoustic rhinometry. Eur Arch Otorhinolaryngol 2012;269:2511-7.

23. Hilberg O. Objective measurement of nasal airway dimensions using acoustic rhinometry: methodological and clinical aspects. Allergy 2002;57 Suppl 70:5-39. 24. Straszek SP, Schlünssen V, Sigsgaard T, Pedersen OF. Reference values for acoustic rhinometry in decongested school children and adults: the most sensitive measurement for change in nasal patency. Rhinology 2007;45:36-9.

25. Hilberg O, Pedersen OF. Acoustic rhinometry: recommendations for technical specifications and standard operating procedures. Rhinol Suppl 2000;16:3-17. Erratum in: Rhinol 2001;39:119.

26. André RF1, Vuyk HD, Ahmed A, Graamans K, Nolst Trenité GJ. Correlation between subjective and objective evaluation of the nasal airway. A systematic review of the highest level of evidence. Clin Otolaryngol 2009;34:518-25.

27. Hong $\mathrm{HR}^{1}$, Jang $\mathrm{YJ}^{1}$. Correlation between remnant inferior turbinate volume and symptom severity of empty nose syndrome. Laryngoscope 2016;126:1290-5. 
TABLE I. Patients' characteristics ( $N=98)$.

\begin{tabular}{ll}
\hline $\begin{array}{l}\text { Age, median (range) } \\
\text { Sex }\end{array}$ & 46 (19-69) \\
Male, No & 56 \\
Female, No & 42 \\
Group, No & \\
Placebo & 14 \\
$\quad$ Allergic sensitization & \\
$\quad$ Yes, No (\%) & $6(43)$ \\
$\quad$ No, No (\%) & $8(57)$ \\
RFA & 28 \\
Allergic sensitization & \\
$\quad$ Yes, No (\%) & $12(43)$ \\
$\quad$ No, No (\%) & $16(57)$ \\
Diode laser & 28 \\
Allergic sensitization & \\
Yes, No (\%) & $12(43)$ \\
$\quad$ No, No (\%) & $16(57)$ \\
MAIT & 28 \\
Allergic sensitization & \\
Yes, No (\%) & $13(46)$ \\
No, No (\%) & $15(54)$ \\
\hline \hline
\end{tabular}


TABLE II. Comparison of operation time, pain during the operation, and bleeding of the groups.

\begin{tabular}{|c|c|c|c|c|c|}
\hline & Time (s $(95 \% \mathrm{Cl}))$ & Pain (VAS $(95 \% \mathrm{CI}))$ & $\begin{array}{l}\text { Nasal packing } \\
\text { needed (No) }\end{array}$ & $\begin{array}{l}\text { Post-operative } \\
\text { bleeding (No) }\end{array}$ & $\begin{array}{l}\text { Post-operative } \\
\text { bleeding requiring } \\
\text { treatment (No) }\end{array}$ \\
\hline \multicolumn{6}{|l|}{ Group } \\
\hline Placebo & 186 (167-205) & $1.4(0.6-2.1)$ & 0 & 0 & 0 \\
\hline RFA & 217 (194-240) & $2.4(1.6-3.1)$ & 0 & 1 & 0 \\
\hline Diode laser & $374(329-420)$ & $2.3(1.6-3.1)$ & 1 & 0 & 0 \\
\hline MAIT & $545(508-583)$ & $2.9(2.1-3.6)$ & 5 & 4 & 3 \\
\hline
\end{tabular}


TABLE III. Change in the VAS scores for nasal symptoms at three months.

\begin{tabular}{|c|c|c|c|c|c|}
\hline & Placebo & RFA & Diode laser & MAIT & $p$-value + \\
\hline \multicolumn{6}{|l|}{$\begin{array}{l}\text { Severity of obstruction, } \\
\text { median (IQR) }\end{array}$} \\
\hline Preoperative & $7.0(5.8-8.0)$ & $8.0(6.3-8.9)$ & $8.0(7.0-8.0)$ & $8.0(7.0-9.0)$ & NS \\
\hline Post-operative & $4.0(2.0-6.3)$ & $2.0(1.0-3.8)$ & $3.0(1.6-4.0)$ & $3.0(1.6-4.0)$ & \\
\hline Change, median & $-2.5(-5.0--0.8)$ & $-5.0(-7.0--3.3)$ & $-5.0(-6.0--3.0)$ & $-5.5(-6.4--3.0)$ & $0.04 *$ \\
\hline Change, mean $(95 \% \mathrm{Cl})$ & $-2.6(-4.0--1.3)$ & $-4.7(-5.6--3.8)$ & $-4.7(-5.4--4.0)$ & $-4.7(-5.7--3.7)$ & \\
\hline$p$-value $\ddagger$ & $0.004 *$ & $<0.001^{*}$ & $<0.001 *$ & $<0.001 *$ & \\
\hline \multicolumn{6}{|l|}{ Change compared to } \\
\hline$p$-value $\S$ & & $0.03^{*}$ & $0.02 *$ & $0.04^{*}$ & \\
\hline \multicolumn{6}{|l|}{ Discharge, median (IQR) } \\
\hline Preoperative & $3.5(1.8-5.3)$ & $6.0(2.3-7.8)$ & $5.5(3.3-7.0)$ & $5.0(3.3-7.0)$ & NS \\
\hline Post-operative & $2.0(0.9-3.0)$ & $2.0(0.0-4.8)$ & $2.0(1.0-4.1)$ & $2.0(1.0-4.5)$ & \\
\hline Change, median & $-1.5(-4.0--0.3)$ & $-2.0(-5.0--0.6)$ & $-2.5(-4.0--1.0)$ & $-2.0(-4.8--0.4)$ & NS \\
\hline$p$-value $\ddagger$ & 0.06 & $<0.001 *$ & $<0.001 *$ & $0.001 *$ & \\
\hline \multicolumn{6}{|l|}{ Crust, median (IQR) } \\
\hline Preoperative & $4.6(3.0-6.3)$ & $5.4(4.6-6.3)$ & $5.3(4.6-6.0)$ & $6.0(5.1-6.8)$ & NS \\
\hline Post-operative & $3.1(1.6-4.6)$ & $3.0(2.1-3.8)$ & $3.3(2.4-4.3)$ & $3.0(2.1-4.0)$ & \\
\hline Change, median & $-2.0(-3.3--0.4)$ & $-2.0(-5.0--1.0)$ & $-2.0(-3.8-0.0)$ & $-3.0(-4.0--1.3)$ & NS \\
\hline$p$-value $\ddagger$ & 0.08 & $<0.001^{*}$ & $<0.001 *$ & $<0.001 *$ & \\
\hline
\end{tabular}

The distribution of the data is non-parametric, hence medians and interquartile ranges (IQR: Q25-Q75) are used in the statistical testing. + Kruskal-Wallis test; $¥$ Wilcoxon signed-rank test; § Mann-Whitney $U$ test with Bonferroni correction; NS = not significant; ${ }^{*}$ statistically significant 
TABLE IV. Change in V2-5 cm acoustic rhinometry values (one side of the nasal cavity).

\begin{tabular}{|c|c|c|c|c|c|}
\hline & Placebo & RFA & Diode laser & MAIT & $p$-valuet \\
\hline \multicolumn{6}{|l|}{$\mathrm{V} 2-5 \mathrm{~cm}\left(\mathrm{~cm}^{3}\right)$} \\
\hline \multicolumn{6}{|l|}{ Preoperative } \\
\hline Median (IQR) & $3.60(2.82-4.94)$ & $4.39(3.02-5.65)$ & $3.47(2.714 .36)$ & $3.57(2.52-4.58)$ & NS \\
\hline \multicolumn{6}{|l|}{ Post-operative } \\
\hline Median (IQR) & $3.94(2.82-5.33)$ & 5.39 (4.29-6.48) & $4.37(3.56-5.60)$ & $4.27(3.37-5.41$ & \\
\hline \multicolumn{6}{|l|}{ Change } \\
\hline Median (IQR) & $0.39(-0.21-0.96)$ & $1.12(-0.38-2.39)$ & $0.84(-0.41-1.66)$ & $0.64(-0.27-1.56)$ & NS \\
\hline Mean $(95 \% \mathrm{Cl})$ & $0.13(-0.51-0.77)$ & $1.14(0.51-1.78)$ & $0.85(0.43-1.26)$ & $0.86(0.30-1.41)$ & \\
\hline$p$-value $\ddagger$ & NS & $<0.001^{*}$ & $0.001 *$ & $0.003^{*}$ & \\
\hline
\end{tabular}

The distribution of the data is non-parametric, hence medians and interquartile ranges (IQR: Q25-Q75) are used in the statistical testing. + Kruskal-Wallis test; ¥ Wilcoxon signed-rank test; NS = not significant; * statistically significant 
Figure I. Mean changes in the severity of nasal obstruction (A), discharge (B), crust (C) and pain (D) after the operation.
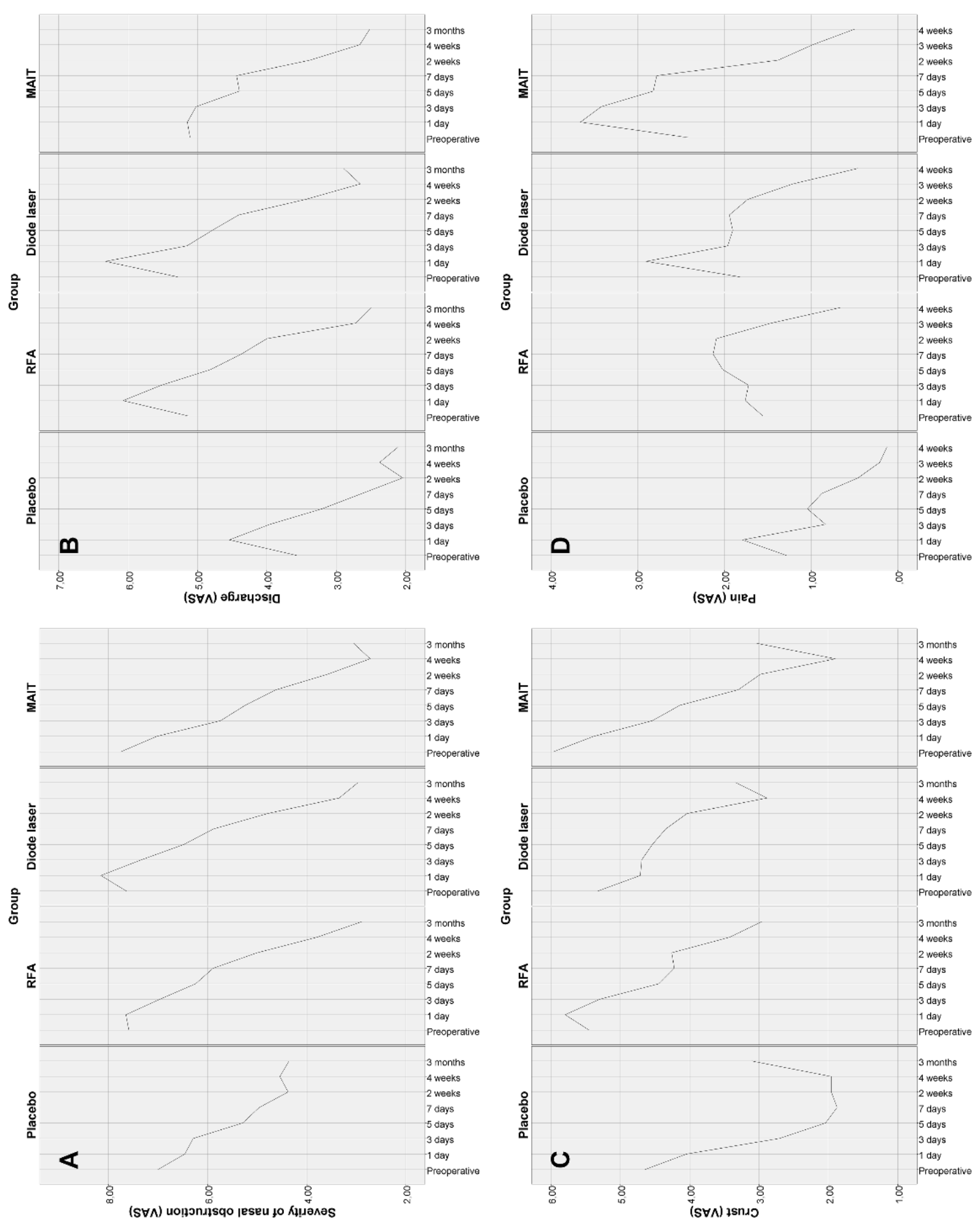
